\title{
Quantum criticality and universality in the $p$-wave paired Aubry-André-Harper model
}

\author{
Ting Lv, ${ }^{1,2}$ Tian-Cheng Yi, ${ }^{3}$ Liangsheng Li ${ }^{4}$ Gaoyong Sun,,${ }^{1,2}$ and Wen-Long You ${ }^{1,2, *}$ \\ ${ }^{1}$ College of Science, Nanjing University of Aeronautics and Astronautics, Nanjing, 211106, China \\ ${ }^{2}$ Key Laboratory of Aerospace Information Materials and Physics (NUAA), MIIT, Nanjing 211106, China \\ ${ }^{3}$ Beijing Computational Science Research Center, Beijing 100193, China \\ ${ }^{4}$ Science and Technology on Electromagnetic Scattering Laboratory, Beijing 100854, China
}

(Dated: January 12, 2022)

\begin{abstract}
We investigate the quantum criticality and universality in Aubry-André-Harper (AAH) model with $p$-wave superconducting pairing $\Delta$ in terms of the generalized fidelity susceptibility (GFS). We show that the higher-order GFS is more efficient in spotlighting the critical points than lowerorder ones, and thus the enhanced sensitivity is propitious for extracting the associated universal information from the finite-size scaling in quasiperiodic systems. The GFS obeys power-law scaling for localization transitions and thus scaling properties of the GFS provide compelling values of critical exponents. Specifically, we demonstrate that the fixed modulation phase $\phi=\pi$ alleviates the odd-even effect of scaling functions across the Aubry-André transition with $\Delta=0$, while the scaling functions for odd and even numbers of system sizes with a finite $\Delta$ cannot coincide irrespective of the value of $\phi$. A thorough numerical analysis with odd number of system sizes reveals the correlationlength exponent $\nu \simeq 1.000$ and the dynamical exponent $z \simeq 1.388$ for transitions from the critical phase to the localized phase, suggesting the unusual universality class of localization transitions in the AAH model with a finite $p$-wave superconducting pairing lies in a different universality class from the Aubry-André transition. The results may be testified in near term state-of-the-art experimental settings.
\end{abstract}

\section{INTRODUCTION}

Quantum phase transitions (QPTs) have attracted the intense interest of both theorists and experimentalists in condensed matter physics for decades. With variation of a non-thermal variable in the many-body Hamiltonian, the ground-state properties show abrupt changes as a result of competing ground-state phases [1-3]. The Landau-Ginzburg-Wilson (LGW) paradigm has provided a well-established framework, which rests on the assumption that a continuous equilibrium phase transition can be accessed by the onset of an order parameter associated with spontaneous symmetry breaking. A broad regime of order parameter fluctuations are controlled by proximity to a quantum critical point (QCP). It has been a long time to recognize that the symmetry breaking occurs spontaneously only in infinite-size quantum many-body systems, while recently they are sometimes observed in surprisingly small systems [4] and even few-body systems [5-7]. Despite the enormous success, the breakdown of the LGW paradigm appears in a few different situations, for example, deconfined QCPs [8, 9] and deconfined critical universality classes [10].

In recent years, an immense effort has been expended to understand QPTs in quasiperiodic systems [11-22]. The quasiperiodic system possesses a long-range periodicity, which is intermediate between that of the clean and randomly disordered cases, offering a rich playground to study quasiperiodic QCPs and unusual characteristic features, such as hierarchical energy spectra [23-25] and localization-delocalization transitions. A paradigmatic

*wlyou@nuaa.edu.cn model of the quasiperiodic system is the Aubry-AndréHarper (AAH) model [26-45], in which the quasiperiodicity is embodied in the form of a cosine modulation incommensurate with lattice spacing. With the rapid development of experimental technologies, the AAH model can be realized in optical waveguide lattices [46-48], photonic crystals [49-51] and cold atom systems [52]. For instance, the AAH Hamiltonian has been experimentally realized by cold atomic gases in a one-dimensional optical lattice perturbed by another weak incommensurate optical lattice [52]. These feasible platforms allow us to explore the emerging topological states of matter with additional interactions in incommensurate systems, including modulated off-diagonal hopping [29], nearest neighbor $p$-wave superconductivity [53], a long-range $p$-wave superconducting pairing [34], and many-body interactions $[38,54]$.

The AAH model has gained popularity since it acts as a proxy for random potentials in the study of generic disordered system. An obstacle to comprehending the critical phenomena in the disordered systems is the undecidability of local order parameters. In close proximity to QCPs, the complex and non-local entanglement between individual constituents becomes extremely prominent at all distance scales. As such, it has been recognized that the exploration of quantum critical phenomena from the perspective of quantum information science is a great privilege, such as the von Neumann entropy [55], and quantum concurrence [56]. The quantum fidelity susceptibility (QFS) has proved to be particularly useful for detecting the critical points of a symmetry-knowledge unknown system [57-60]. It was shown that the QFS can not only identify the QCPs, but also satisfy the scaling ansatz, where the universal information can be retrieved. The 
most significant implication is that the finite-size scaling of such a universal order parameter dictates position of QCPs and the critical exponent of the correlation length $\nu$. For a quasi-periodic system with spatial complexity, the scaling theory of the QFS and the universality of localization transition have been partially understood. Notably, the finite-size scaling of the usual fidelity susceptibility is irrelevant to the dynamical exponent $z$. However, the critical exponents obey the scaling and hyperscaling relations, implying that there are only two independent exponents. Thus, a second independent critical exponent plays a decisive role in determining the universality class, which lies at the heart of critical phenomena. In this work, we apply the generalization of fidelity susceptibility to the one-dimensional AAH model with $p$-wave superconducting pairing, and devise a direct pathway to the determination of critical points and universal critical exponents of localization-delocalization transitions. Importantly, the theoretical predictions could be testified in state-of-the-art experiments.

The rest of the paper is organized as follows. Section II reviews the AAH model with $p$-wave superconductivity and determines its phase diagram. In Sec. III, we introduce the concept of the generalized fidelity susceptibility (GFS) and postulate its scaling hypothesis for the universal part. Section IV is devoted to the scaling behavior of the GFS in the AAH model and identification of critical exponents. Conclusions and discussions are presented in Sec. V.

\section{MODEL HAMILTONIAN}

The generalized AAH model with $p$-wave superconducting pairing in a quasi-periodically modulated potential is given by the following Hamiltonian:

$$
H=\sum_{j=1}^{N}\left(-J c_{j}^{\dagger} c_{j+1}+\triangle c_{j} c_{j+1}+\text { H.c }\right)+\sum_{j=1}^{N} V_{j} c_{j}^{\dagger} c_{j},(1)
$$

where $c_{j}^{\dagger}\left(c_{j}\right)$ is the fermionic creation (annihilation) operator at the $j$-th site among total $N$ lattice sites, $J$ is the hopping strength between nearest-neighbor sites, $\Delta$ denotes the amplitude of $p$-wave superconducting pairing, and H.c. represents the Hermitian conjugate. The $p$-wave pairing amplitudes can be tuned by the mixture of spin-polarized fermions with a Bose-Einstein condensate [61], affected by an $s$-wave Feshbach resonance in a spin-polarized cold Fermi gas [62], or induced by the proximity effect in stacking a superconducting wire on top of the normal metal. Here we focus on quasiperiodicity encoded in the chemical potential, keeping a constant hopping magnitude and pairing potential. The on-site potential terms are quasiperiodically varying according to the Aubry-André rule $V_{j}=V \cos (2 \pi \alpha j+\phi)$, where $\alpha=(\sqrt{5}-1) / 2$ is an irrational frequency and $V$ is the strength of the incommensurate potential. The parameter $\phi \in[0,2 \pi)$ shifts the origin of the modulation representing a random phase. The boundary condition is imposed as $c_{N+1}=\sigma c_{1}$, where $\sigma=1,-1$, and 0 corresponding to periodic, antiperiodic, and open boundary conditions, respectively. Without losing generality, $\Delta$ can be assumed to be real the phase can be otherwise eliminated under global U(1) transformation] and $J=1$ is set as energy unit throughout the paper. For $\Delta= \pm 1$, the model will be equivalent to quasiperiodic Ising model $[63,64]$. When the $p$-wave pairing term is absent, i.e., $\Delta=0$, the AAH model in Eq.(1) becomes easily tractable as it can be written as $H=\sum_{i, j} c_{i}^{\dagger} \mathcal{H}_{i, j} c_{j}$. The eigenvectors $\left|\psi_{n}\right\rangle$ and the associated single-particle energies $\epsilon_{n}$ are obtained by diagonalizing the $N \times N$ singleparticle Hamiltonian matrix $\mathcal{H}$. In the limit when $V / J \rightarrow$ 0 , Eq. (1) describes a metallic chain with all eigenstates being extended, while for $V / J \rightarrow \infty$ the eigenmodes are localized on one site. The Aubry-André transition from being extended to being localized is known to occur at $V / J=2$ as a consequence of Aubry-André duality between the Hamiltonian in position and momentum space.

As for a finite $p$-wave paring, i.e., $\Delta \neq 0$, the Hamiltonian (1) can be diagonalized through a canonical Bogoliubov-de Gennes (BdG) transformation by introducing the new fermionic operators $\eta_{n}$ and $\eta_{n}^{\dagger}$,

$$
\eta_{n}=\sum_{j=1}^{N}\left(u_{n, j}^{*} c_{j}+v_{n, j} c_{j}^{\dagger}\right), \quad c_{j}=\sum_{n=1}^{N}\left(u_{n, j} \eta_{n}+v_{n, j}^{*} \eta_{n}^{\dagger}\right),
$$

where $u_{n, j}$ and $v_{n, j}$ denote the two components of the wave function at site $j$, and $n(n=1, \ldots, N)$ is the energy band index. The eigenstates $\left|\psi_{n}\right\rangle=$ $\left(u_{n, 1}, u_{n, 2}, \ldots, v_{n, 1}, v_{n, 2}, \ldots\right)^{T}$ can be determined by solving the Schrödinger equation $H\left|\psi_{n}\right\rangle=E_{n}\left|\psi_{n}\right\rangle$, which can be recast into a $2 N \times 2 N$ matrix form as

$$
\left(\begin{array}{cc}
A & B \\
-B^{*} & -A^{T}
\end{array}\right)\left(\begin{array}{c}
u_{n, i} \\
v_{n, i}^{*}
\end{array}\right)=\epsilon_{n}\left(\begin{array}{c}
u_{n, i} \\
v_{n, i}^{*}
\end{array}\right),
$$

where $A(B)$ is a $N \times N$ symmetric (antisymmetric) matrix. The nonzero elements are given by $A_{i, i}=V_{i}, A_{i, i+1}=$ $A_{i+1, i}=-J$, and $B_{i, i+1}=-B_{i+1, i}=\Delta$. The matrix elements for the boundary terms are $A_{N, 1}=A_{1, N}=-\sigma J$, and $B_{N, 1}=-B_{1, N}=-\sigma \Delta$. The BdG Hamiltonian $\mathcal{H}$ in Eq.(3) respects an imposed particle-hole symmetry, namely, $\tau^{x} \mathcal{H}^{T} \tau^{x}=-\mathcal{H}$, where the Pauli matrix $\tau^{x}$ acts in the Nambu space. The energy levels appear in $\pm \epsilon_{n}$ conjugate pairs, with $\epsilon_{n} \geq 0$, except the zero energy mode, which is self-conjugate. As such, for finite lattices it is convenient to replace $\alpha$ with $F_{\ell-1} / F_{\ell}$, the ratio of two successive Fibonacci numbers $[65,66]$. Note that the irrational limit is reached as far as the numerical results are extrapolated to the scaling limit $(\ell \rightarrow \infty)$. The period $F_{\ell}$ then acts like a finite length scale which controls scaling behavior. The Fibonacci-sequence quasiperiodic potential has an intimate connection with topological phase transition and Majorana modes.

While in the translational-invariant case the solution of Eq.(3) can be further reduced to the $2 \times 2$ matrix form with independent momenta, in the quasidisorder case one 


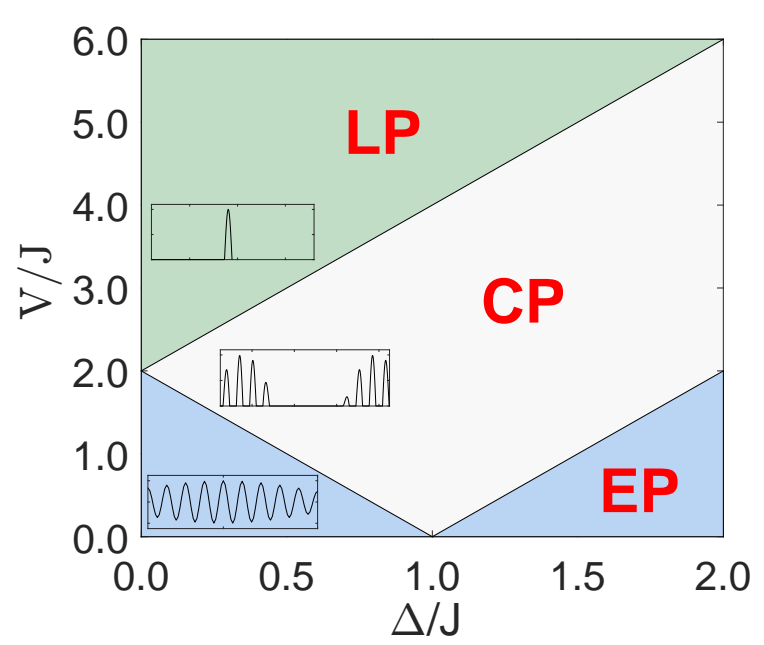

FIG. 1. Phase diagram of the AAH model as a function of $p$-wave pairing strength $\Delta$ and incommensurate potential strength $V$. The localized phase (LP), critical phase (CP), and extended phase (EP) are marked by blue, white, and green respectively. Three insets show the typical spatial distribution for localized, critical, and extended modes.

has to diagonalize the $2 N \times 2 N$ BdG matrix numerically, marking a qualitative difference between the disordered and the clean model. In terms of the new fermion operators, the Hamiltonian in Eq.(1) can be diagonalized as

$$
H=\sum_{n=1}^{N} \epsilon_{n} \eta_{n}^{\dagger} \eta_{n}-\epsilon_{n} \eta_{n} \eta_{n}^{\dagger}=\sum_{n=1}^{N} 2 \epsilon_{n}\left(\eta_{n}^{\dagger} \eta_{n}-\frac{1}{2}\right),
$$

with the single-particle eigenvalues being $\epsilon_{1} \leq \epsilon_{2} \leq \ldots$ $\leq \epsilon_{N}$. The ground state of $H$ is the Bogoliubov vacuum state $\left|\psi_{g}\right\rangle$ annihilated by all $\eta_{n}$ for $n=1, \ldots, N$, i.e., $\eta_{n}\left|\psi_{g}\right\rangle=0$, with an energy $E_{g}=-\sum_{n=1}^{N} \epsilon_{n}$. For a weak quasi-disorder strength, all the eigenstates of the system are extended, while the system becomes localized for a sufficiently strong disorder. Recently, it was found that with a nonzero superconducting pairing, a nonergodic critical phase intervenes the transition from the delocalized to localized state, and all the eigenstates are expected to be multifractal. The phase diagram shown in Fig. 1 consists of the extended phase (EP), critical phase (CP), and localized phase (LP). The localized wavefunctions in the LP can be transformed into the extended ones in the EP by a Aubry-André duality occurring at $\Delta=0$ across a second-order QCP. The system undergoes a continuous QPT from the EP to the CP at $V_{c 1}=2|J-\Delta|$. One finds that Eq.(1) is invariant under the transformation as $c_{j} \rightarrow-c_{j}^{\dagger}$ on odd $j$-th sites and $\alpha \rightarrow \alpha+1 / 2$ [36]. For the self-duality point $(J=\Delta, V=0)$, the EP becomes unstable for arbitrarily weak disorder. The system displays a second-order QPT from $\mathrm{CP}$ to LP at $V_{c 2}=2|J+\Delta|$.

In order to visually characterize the localized and extended nature of the entire energy spectrum, we evaluate
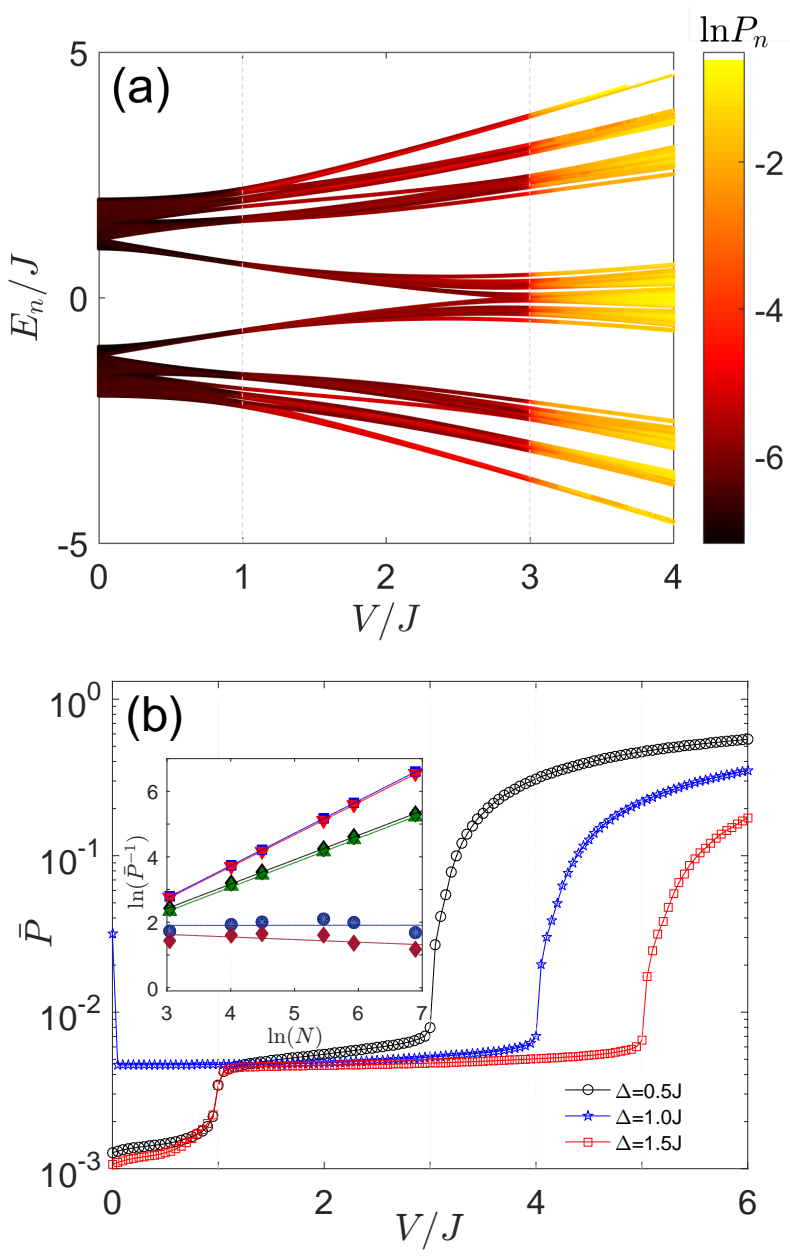

FIG. 2. (a) Normalized IPR on a logarithmic scale for all eigenmodes of Eq. (1) as a function of eigenenergies and $V / J$ with $\Delta=0.5, N=987$. The dashed lines mark the critical points at $V_{c 1}=2|J-\Delta|$ and $V_{c 2}=2|J+\Delta|$, respectively. The logarithmic scale is shown to have a better resolution. (b) MIPR as a function of the quasi-disorder strength at $\Delta=$ $0.5 J$ (black circles), $\Delta=J$ (blue pentagons), and $\Delta=1.5 J$ (red squares). Here we use the phase $\phi=\pi$ and the lattice site $N=987$. Inset shows the finite-size scaling of $\bar{P}^{-1}$ for $V=0.2 J, 0.5 J, 2.0 J, 2.5 J, 3.5 J$, and $4.0 J$ for $\Delta=0.5 J$.

the normalized inverse participation ratio (IPR) for each eigenstate $\left|\psi_{n}\right\rangle$ of the model [67-70], given by

$$
P_{n}=\frac{\sum_{j}\left(u_{n, j}^{4}+v_{n, j}^{4}\right)}{\sum_{j}\left(u_{n, j}^{2}+v_{n, j}^{2}\right)} .
$$

The IPRs can quantify the extent of distribution over the preferential bases. It should be noted that IPR is erroneously employed to describe the participation ratio in many literatures reports $[18,71]$, which is the reciprocal of IPR [72]. In a specific $\mathcal{N}$-dimensional bases $\left|\varphi_{k}\right\rangle$, the IPR of the state $\left|\psi_{n}\right\rangle=\sum_{k=1}^{\mathcal{N}} c_{k}\left|\varphi_{k}\right\rangle$ reaches a maximal value $P_{\max }=1$ when the state coincides exactly a single basis state, and attains a minimal value $P_{\min }=1 / \mathcal{N}$ when the state is uniform in the selective bases. For a 

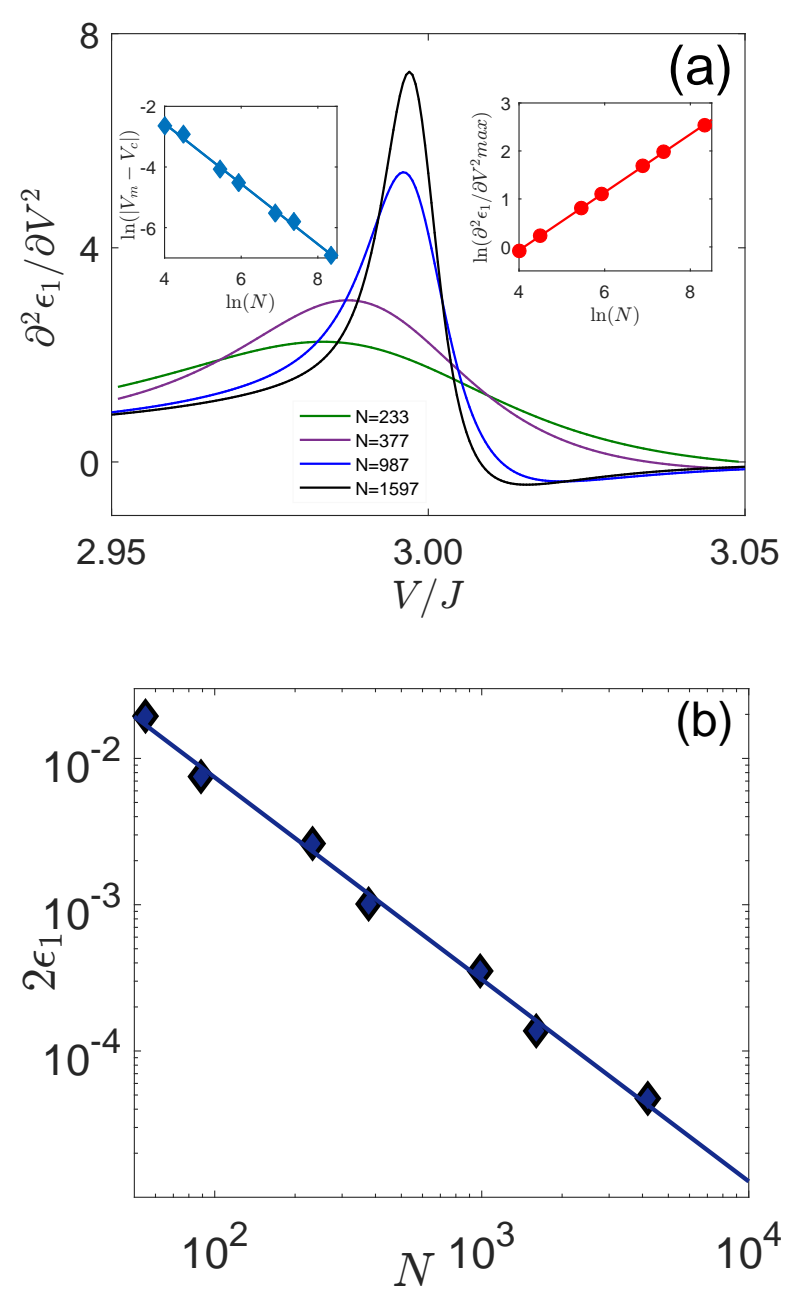

FIG. 3. (a) The second-order derivative of first excitation $\epsilon_{1}$ with respect to $V$. Inset shows the scaling in the vicinity of the critical point $\ln \left(\left|V_{m}-V_{c}\right|\right)=-0.994( \pm 0.066)$ $\ln N+1.408( \pm 0.410)$ and the scaling behavior between the maximum value of $\ln \left(\partial^{2} \epsilon_{1} / \partial V^{2}\right)_{\max }=0.605( \pm 0.010) \ln N$ $-2.491( \pm 0.063)$. The symbols in the insets denote the numerical results and the solid lines correspond to the linear fittings. The parameters are $\Delta=0.5, \phi=\pi$. (b) The excitation gap $2 \epsilon_{1}$ around the critical point $V_{c 2}=3$ as a function of $N$ with $\Delta=0.5, \phi=\pi$.

set of one-particle states in real space, the IPR scales inversely with the system size $N$ in the delocalized state, while appears to be independent of $N$ in the localized phase and shows intermediate behavior in the CP. Both transitions at finite strength of quasiperiodic modulation occur simultaneously for all eigenstates, as is revealed in Fig.2(a), in contrast to the presence of mobility edges in specific systems [73-76]. We thus further define the mean inverse participation ratio (MIPR) as

$$
\bar{P}=\sum_{n=1}^{2 N} \frac{1}{2 N} P_{n} .
$$

The evolution of MIPR is exhibited in Fig.2(b) on a logarithmic scale for different $p$-wave pairing strength $\Delta=0.5 \mathrm{~J}, \Delta=1.0 \mathrm{~J}$, and $\Delta=1.5 \mathrm{~J}$. One finds the MIPR is capable of identifying the phase boundaries separating the extended, critical, and localized phases, which are captured by the turning points of the MIPR locating respectively at $V_{c 1}=2|J-\Delta|$ and $V_{c 2}=2|J+\Delta|$. The LP is gapless for periodic boundary conditions while gapped for open boundary conditions. Note that for a pure state $\rho=\left|\psi_{n}\right\rangle\left\langle\psi_{n}\right|$ of the entire system, the von Neumann entropy is zero and the IPR is inversely proportional to the participation entropy $S_{q}=\ln \sum_{k} \rho_{k k}^{q} /(1-q)$ of order $q=2$, i.e., $S_{2}=-\ln P$, which becomes the diagonal entropy for the reduced density matrix of the subsystem [77, 78]. As is observed in the inset of Fig.2(b), the scaling exponents extracted from the linear fittings for $V=0.2 J$ and $V=0.5 J$ with $\Delta=0.5 J$ are approximately 0.98, which implies that the inverse of MIPR tends to scale extensively for extended states in the AAH model, resembling the volume-law scaling of the mean first-order Rényi entropy at infinite temperature, which is conjectured to be universal for translationally invariant quadratic fermionic Hamiltonians [79], while $\bar{P}^{-1}$ declines towards a finite value close to $\mathcal{O}(1)$ for localized states, in analogy to the area law of the disordered averaged entanglement entropy [80]. For critical states, the MIPR scales like $N^{-d^{*}}$ [81], where the fractal dimension $0<d^{*}<1$ depends on the fractal structure of wavefunctions. The fitting lines of $\ln \left(\bar{P}^{-1}\right)$ with respect to $\ln N$ for $V=1.5 \mathrm{~J}$ and $V=2.0 \mathrm{~J}$ with $\Delta=0.5 \mathrm{~J}$ gives rise to $d^{*} \approx 0.75$, implying that points in the whole CP belong to the same universality class. Remarkably, the fractal dimension $d^{*}$ and the associated IPR are proved to host intrinsic relation to the mean entanglement entropy [82]. One should be aware that a typical value of IPR can be used $\bar{P}_{\text {typ }}=\sum_{n=1}^{2 N} \ln P_{n} /(2 N)$, which is similar to the behavior of Eq.(6) for the AAH model (1) yet becomes more subtle when eigenstates display a single-particle mobility edge.

\section{GENERALIZED FIDELITY SUSCEPTIBILITY AND SCALING HYPOTHESIS}

It is now well established that the QFS is a good measure to witness QPTs and manifest critical phenomena in translational invariant quantum systems [83-85]. The merit of the QFS in characterizing critical phenomena is the model-independent feature, which is quite suitable for quantum systems without prior knowledge of order parameters. To this end, the fidelity susceptibility is recognized as a sensitive probe of quantum criticalities in conjugate field [86], long-range interacting systems [87, 88], deconfined QCP [89], disordered systems, chaotic Hamiltonians [90], quantum many-body scars [91], excited-state quantum phase transition [92], and holographic models [93]. Currently the investigations of the scaling of QFS in the context of quantum 
disordered systems are still poorly understood. In what follows, we will focus on the fidelity susceptibility and its generalization as well as the associated scaling in the AAH model.

The fidelity susceptibility provides a generic and direct approach to measure the quantum metric tensor via the transition probability of the quantum state being excited to other eigenstates during a sudden infinitesimal quench of the tuning parameter $\lambda$ [85]. The GFS of order $2 r+2$ at the tuning parameter $\lambda$ associated with the state $\left|\psi_{n}(\lambda)\right\rangle$ is given by [94]

$$
\chi_{2 r+2}^{(n)}(\lambda)=\sum_{m \neq n} \frac{\left|\left\langle\psi_{m}(\lambda)\left|\partial_{\lambda} \hat{H}\right| \psi_{n}(\lambda)\right\rangle\right|^{2}}{\left[E_{m}(\lambda)-E_{n}(\lambda)\right]^{2 r+2}},
$$

where $\left|\psi_{m}(\lambda)\right\rangle$ and $E_{m}(\lambda)$ correspond to the $m$ th eigenstate and eigenvalue of this generic Hamiltonian $\hat{H}(\lambda)$, respectively. The numerator in Eq.(7) denotes the probability of exciting the system away from the state $\left|\psi_{n}(\lambda)\right\rangle$ through a relevant (or marginal) perturbation $\partial_{\lambda} \hat{H}$. The GFS of different orders is embodied by the power of the denominator. Concretely, Eq.(7) reduces respectively to the second derivative of the ground-state energy $\chi_{1}$ for $r=-1 / 2$ [59] and the conventional quantum geometric tensor $\chi_{2}$ for $r=0$ [85]. We can anticipate that $\chi_{1}$ has a weaker divergence than $\chi_{2}$ at a critical point. The QFS can be also devised as the Riemannian metric tensor upon projecting the dynamics onto a single (non-degenerate) band [95],

$$
\chi_{2}^{(n)}(\lambda)=\left\langle\partial_{\lambda} \psi_{n}\left|\left(1-\left|\psi_{n}\right\rangle\left\langle\psi_{n}\right|\right)\right| \partial_{\lambda} \psi_{n}\right\rangle
$$

Regarding the absence of mobility edge in the energy spectrum, in the following we focus on the GFS of the lowest eigenstate $\left|\psi_{1}(\lambda)\right\rangle$. To this end, the superscript of $\chi_{2 r+2}^{(1)}(r=-1 / 2,0,1)$ is omitted for abbreviation.

We then start the description of the finite-size scaling theory by recalling its main features that hold in the vicinity of the usual continuous QPT. The sensitivity is greatly enhanced, especially for the system at the quantum criticality compared with that away from the critical region [96]. Single parameter scaling posits that the correlation length $\xi$ is the only relevant length scale in the thermodynamic limit that diverges at the transition,

$$
\xi \sim\left|\lambda-\lambda_{c}\right|^{-\nu},
$$

and the single-particle spectral gap $\epsilon_{s}$ of size $2 \epsilon_{1}$ will vanish as

$$
\epsilon_{s} \sim N^{-z}
$$

where $\nu$ is the correlation length exponent and $z$ is the dynamical critical exponent. For finite chains, the single parameter scaling hypothesis implies that the relevant physical quantities shall depend only of the ratio $\xi / N$, at least in the vicinity of the critical point where $\xi \gg 1$ [97]. As $\lambda$ crosses QCPs adiabatically, the GFS shows a broad peak for a finite system size, signaling the location of pseudocritical points $\lambda_{m}$. With increasing system sizes
$N$, the peaks of GFS become more pronounced and the maximal points of the GFS is expected that

$$
\chi_{2 r+2}\left(\lambda_{m}\right) \sim N^{\mu}
$$

where $\mu=2 / \nu+2 z r$ is the critical adiabatic dimension. For relevant operators $\partial_{\lambda} \hat{H}$ on sufficiently onedimensional large-size systems, i.e., $\nu<2$, the pseudocritical points converge towards the critical points $\lambda_{c}$, satisfying

$$
\left|\lambda_{m}-\lambda_{c}\right| \propto N^{-\theta}
$$

with $\theta=1 / \nu$. Here one should be aware that the shift exponent $\theta$ in Eq.(12) is not necessarily equal to inverse of the correlation-length exponent $\nu$ [98], as it happens to the entanglement witness $[99,100]$. Accordingly, the GFS of a finite system with size $N$ in the neighborhood of a QCP shall obey the universal scaling form [101],

$$
\chi_{2 r+2}(\lambda)=N^{2 / \nu+2 z r} \phi_{r}\left(\left|\lambda-\lambda_{m}\right| N^{1 / \nu}\right),
$$

where $\phi_{r}(x)$ is a regular universal scaling function of the GFS of order $2 r+2$, a priori unknown. Estimates for critical parameters can thus be obtained by plotting the scaled GFS $\left[\chi_{2 r+2}\left(\lambda_{m}\right)-\chi_{2 r+2}(\lambda)\right] / \chi_{2 r+2}(\lambda)$ versus $N^{1 / \nu}\left(\lambda-\lambda_{m}\right)$ by subtly adjusting the values of $\lambda_{m}, \nu$, and $z$ until data collapse is achieved. Alternatively, taking logarithm on both sides of Eqs.(11) and (12),yields

$$
\begin{array}{r}
\ln \left|\lambda_{m}-\lambda_{c}\right| \propto c_{2 r+2} \ln N, \\
\ln \chi_{2 r+2}\left(\lambda_{m}\right) \propto d_{2 r+2} \ln N,
\end{array}
$$

where fitting parameters $c_{2 r+2}$ and $d_{2 r+2}$ can determine the critical exponents $\nu$ and $z$ as

$$
\nu=-1 / c_{2 r+2}, \quad 2 r z=d_{2 r+2}+2 c_{2 r+2} .
$$

As such, we concentrate our attention on the region close to the critical point of localization-delocalization transitions, above which the eigenstates are localized within the finite localization length $\xi$ [102]. Unlike the Aubry-André model with $\Delta=0$, the BdG Hamiltonian (3) for a finite $\Delta$ acts in an enlarged expanded Nambuspinor space [103]. The second-order derivative of first excitation $\chi_{1} \equiv \partial^{2} \epsilon_{1} / \partial V^{2}$ in the neighborhood of $V_{c 2}$ for various odd number of system sizes $N$ with $\Delta=0.5$ is shown in Fig.3(a). One can see that $\chi_{1}$ presents a divergent peak $\chi_{1, \max }$ at $V_{m}$. The linear fittings give rise to parameters $c_{1}=-0.994 \pm 0.066$ and $d_{1}=0.605$ \pm 0.010 . According to Eq.(14), the fitting values imply that $\nu \approx 1.006$ and $z \approx 1.384$. Furthermore, the singleparticle spectral gap $2 \epsilon_{1}$ with several system sizes from $N=55$ up to $N=4181$ are considered with periodic boundary conditions for $\Delta=0.5$ in Fig.3(b), indicating that $z=1.381 \pm 0.109$ according to Eq. (10).

\section{FIDELITY SUSCEPTIBILITY IN THE AUBRY-ANDRÉ-HAPER MODEL WITH $P$-WAVE PAIRING}

We obtain all the eigenenergies and the corresponding wave functions by diagonalizing Eq.(3). The numerical 
results tempt us to evaluate the GFS of the AAH model through Eq.(7). First we recapitulate the finite-size scaling hypothesis of QFS in the Aubry-André model with $\Delta=0$ [37]. Previous work revealed the QFS near the EPLP transition can be separately rescaled onto two different universal curves for even and odd numbers of lattice sites [32], while the retrieved critical exponents are quite close, which implies that two universal scaling functions are not necessary. The logarithm of QFS $\ln \chi_{2}$ of the Aubry-André model as a function of $V$ for $\Delta=0$ with different system sizes is exhibited in Fig.4(a). One can find that the fidelity susceptibility presents a maximum $\chi_{2 \text {,max }}$ at $V_{m}$. With increasing the system sizes, the peaks become more pronounced and $V_{m}$ gets closer to the critical point $V_{c}=2 J$. The maximum value of $\ln \chi_{2}$ against the system size $N$ is displayed in the log-log scale, whose linear fit shows that $\ln \chi_{2, \max }=(2.000 \pm 0.010) \times \ln N$ 5.400, implying $\nu=1.000 \pm 0.005$ according to Eq.(11). This is consistent with the Harris criterion [104], which imposes that $\nu<2$ for phase transitions in the presence of incommensurate modulation. When the rescaled fidelity susceptibility $\left[\chi_{2}\left(V_{m}\right)-\chi_{2}(V)\right] / \chi_{2}(V)$ is plotted as a function of the proper scaling variable $N^{1 / \nu}\left(V-V_{m}\right)$, all curves of distinct chain sizes in the vicinity of $V_{m}$ collapse into a single curve, as is shown in Fig.4(b), which corroborates the estimated critical parameter and the validity of the single-parameter scaling hypothesis (13). In particular the properly chosen value of $\phi=\pi$ much alleviates the odd-even effect.

Next, the QFS in the AAH model with respect to the strength of the incommensurate potential $V$ for odd number of lattice sizes with $\Delta=0.5$ is shown in Fig.5. The QFS exhibits an extensive scaling in the off-critical region. Therefore, the QFS per site $\chi_{2} / N$ appears to be an $N$-independent value. Instead, the QFS shows a stronger dependence on system size around $V_{c 2}=3$, signaling the onset of the QCP in the AAH model. The maximum values $\chi_{2, \max }$ of the fidelity susceptibility near the QCP as a function of $N$ in $\log -\log$ scale are plotted. The superextensive behavior at the pseudocritical point is reflected in the linear fitting $\ln \chi_{2, \max } \propto(2.003 \pm 0.033) \ln N$, whose slope suggests that $\nu=0.999 \pm 0.017$. Meanwhile, the numerical fitting in terms of Eq.(12) yields $\nu=0.947 \pm 0.090$. The accuracy of retrieved $\nu$ from the algebraic law (12) is generally plagued by the precision of numerical calculation.

In order to extract the dynamical exponent $z$ of $\mathrm{CP}$ LP transitions, we further study the finite-size scaling of $\chi_{4}$. One can easily heed that $\chi_{4}$ displays much more divergent peaks than $\chi_{2}$ in the vicinity of $\mathrm{QCP}$ $V_{c 2}=3$, as is disclosed in Fig.6. The linear fittings of the peak maxima $\chi_{4, \max }$ suggests $c_{4}=-1.018 \pm 0.1074$ and $d_{4}=4.773 \pm 0.202$. According to Eq.(15) the extracted values of critical exponents $\nu=0.993 \pm 0.105$ and $z=1.380 \pm 0.053$ for the CP-LP transition with $\Delta=0.5$ agree well with those obtained from the gap scaling [28]. In this vein, we continue to pick the critical exponents $\nu$ and $z$ via the scaling analysis of $\chi_{4}$ as the $p$-wave super-
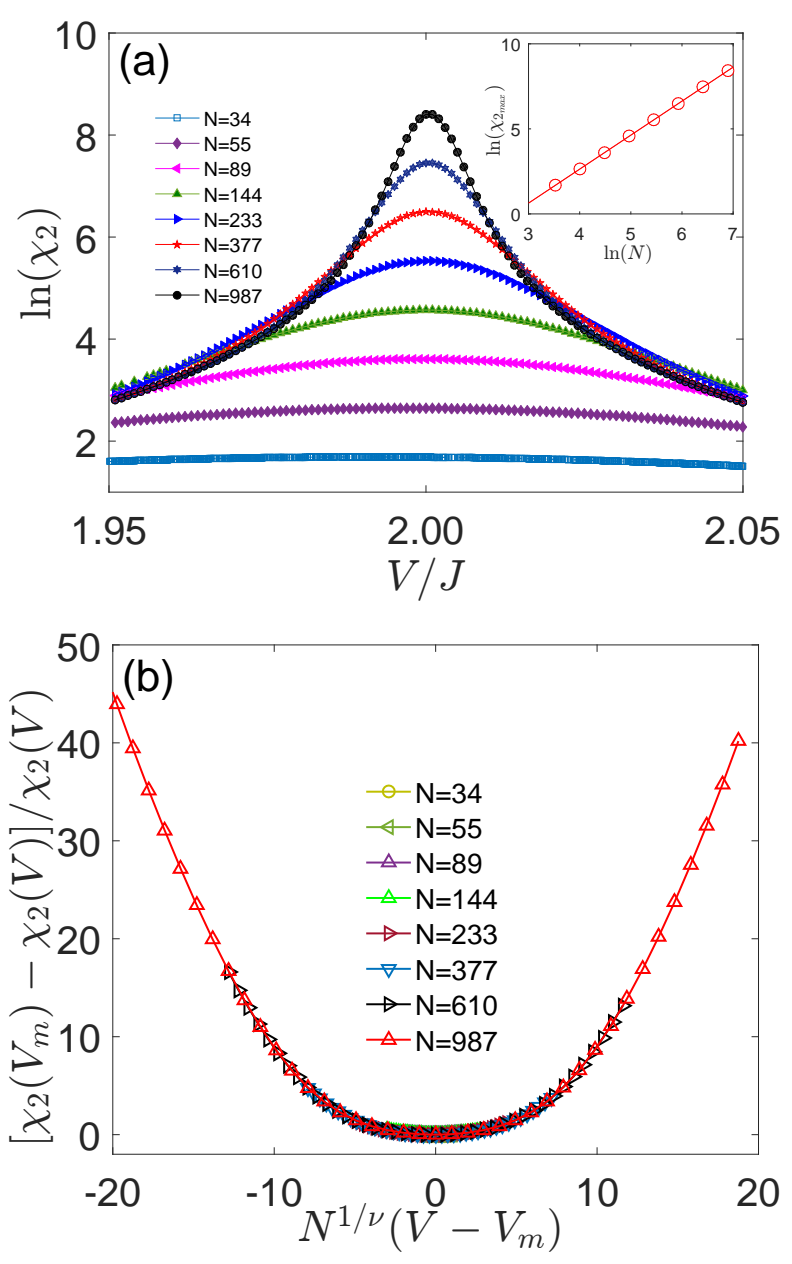

FIG. 4. (a) The logarithm of the fidelity susceptibility $\chi_{2}$ as a function of the strength of the incommensurate potential $V$ for different number of lattice, and the logarithm of the maximum of fidelity susceptibility as a function of the logarithm of the system size, $N=34,55,89,144,233,377,610$, and 987. (b) Scaled fidelity susceptibility $\left[\chi_{2}\left(V_{m}\right)-\chi_{2}(V)\right] / \chi_{2}(V)$ as a function of scaled variable $N^{1 / \nu}\left(V-V_{c}\right)$. All curves for number of the lattice sizes collapse into a single curve when we choose the correlation length critical exponents $\nu=1.000$. Here we choose we take periodic boundary conditions and set $\Delta=0$ and $\phi=\pi$.

conducting pairing $\Delta$ changes. The numerical results in Fig. 7(a) reveals that $\nu \simeq 1.000$ and $z \simeq 1.388$ with little variation. It turns out that there is a discontinuity of $z$ when $\Delta$ increases from 0 to an infinitesimal value. Distinct values of $z \approx 2.375$ for $\Delta=0$ [32] and $z \approx 1.380$ for $\Delta \neq 0$ suggest that their ground states belong to different universality classes. For all $\Delta \neq 0$, the transitions across QCPs $V_{c 2}=2|J+\Delta|$ belong to the same universality class as the quasiperiodic Ising chain [19]. 

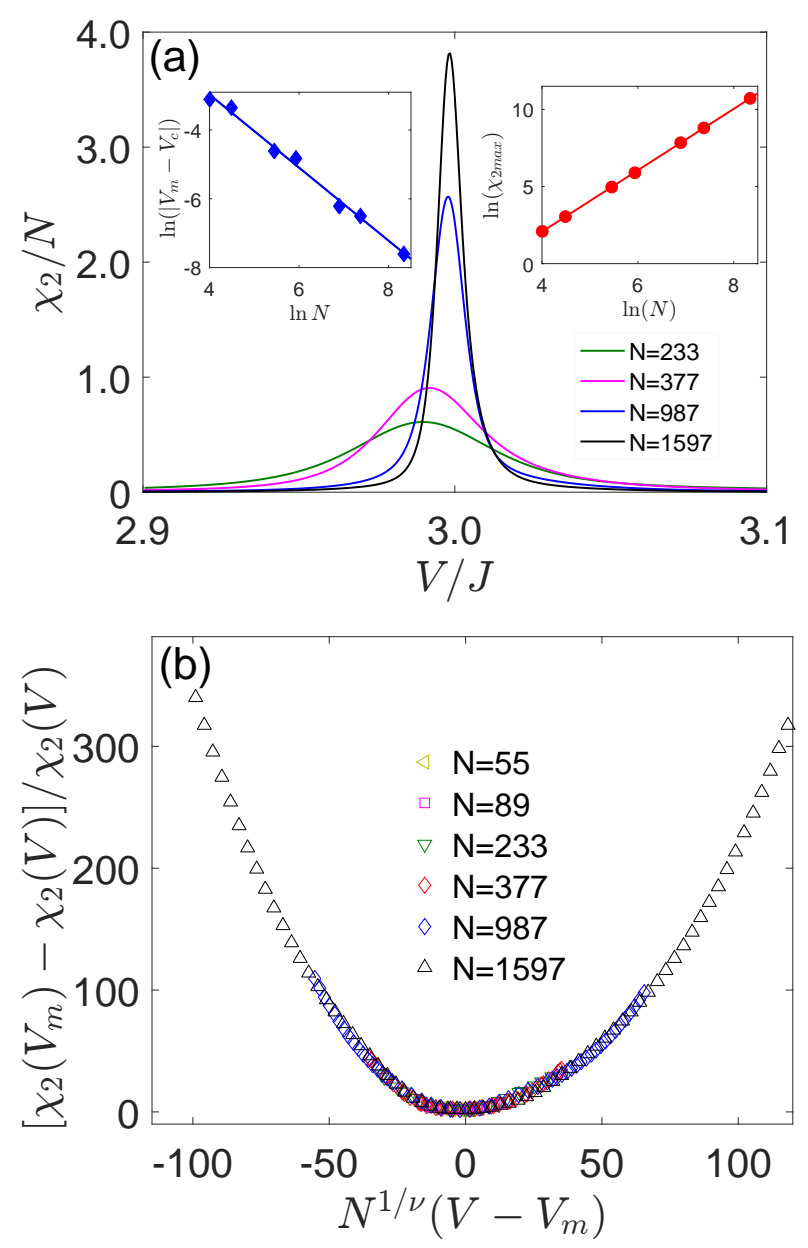

FIG. 5. (a) The fidelity susceptibility per site $\chi_{2} / N$ as a function of the strength of the incommensurate potential $V$ with odd number of lattice sizes around $V_{c 2}=3$. The inset shows the scaling behavior of the maxima versus the system sizes $N=55,89,233,377,987,1597,4181$. (b) Scaled fidelity susceptibility $\left[\chi_{2}\left(V_{m}\right)-\chi_{2}(V)\right] / \chi_{2}(V)$ as a function of scaled variable $N^{1 / \nu}\left(V-V_{m}\right)$. All curves for odd number of the lattice sizes collapse into a single curve when we choose the correlation length critical exponents $\nu=1.00$. Here periodic boundary conditions are used with $\Delta=0.5$ and $\phi=\pi$.

\section{DISCUSSION AND SUMMARY}

In this work, we investigate quantum criticality in the Aubry-André-Harper (AAH) model with $p$-wave superconducting pairing in terms of the generalized fidelity susceptibility (GFS). This quasiperiodic fermion system is Jordan-Wigner-equivalent to the quasi-periodically modulated transverse field $X Y$ chain. The interplay of spatial modulation of potential and symmetry breaking leads to quantum critical phenomena that are different from either the commensurate potential or randomly distributed potential. In the absence of $p$-wave pairing $(\Delta=0)$, the AAH model hosts a phase transition from the extended state to the exponentially localized state
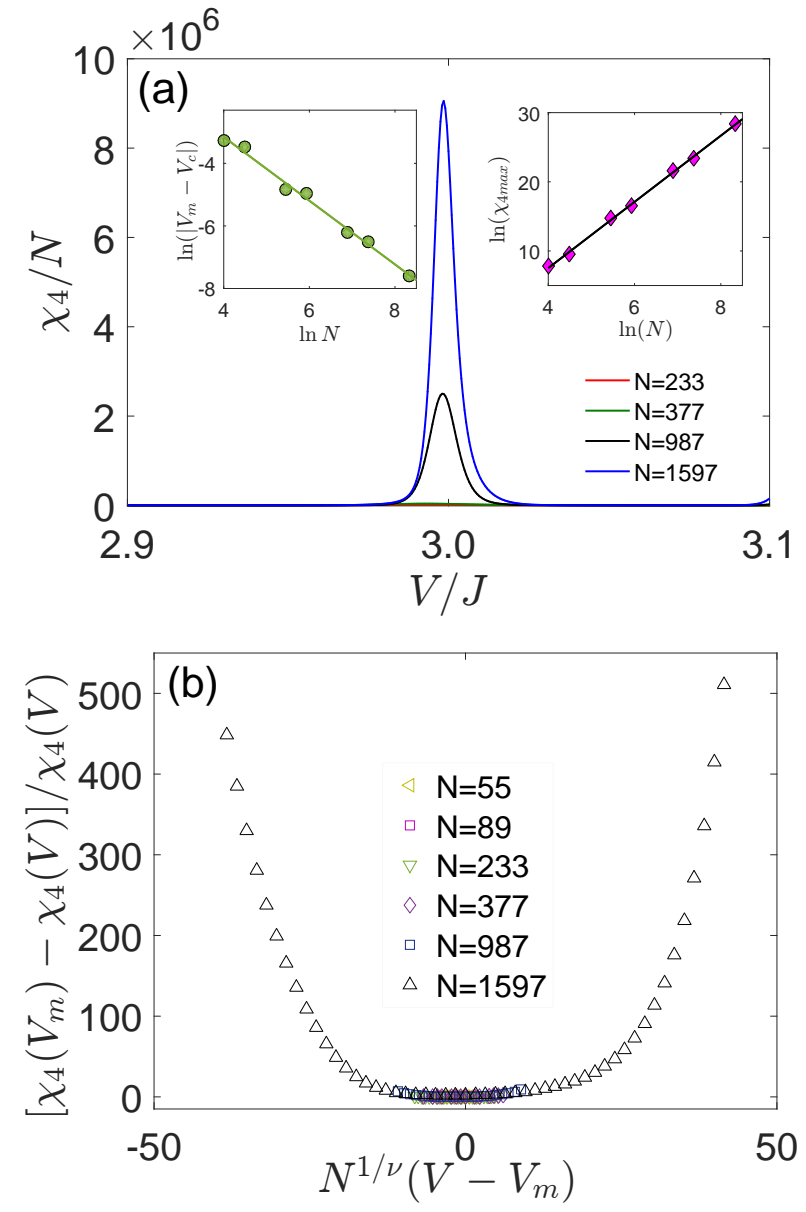

FIG. 6. (a) The GFS per site $\chi_{4} / N$ as a function of the strength of the incommensurate potential $V$ with odd number of lattice sizes around $V_{c 2}=3$. The inset shows the scaling behavior of the maxima versus the system sizes $N=55,89$, 233, 377, 987, 1597, and 4181. (b) Scaled fidelity susceptibility $\left[\chi_{4}\left(V_{m}\right)-\chi_{4}(V)\right] / \chi_{4}(V)$ as a function of scaled variable $N^{1 / \nu}\left(V-V_{m}\right)$. All curves for odd number of the lattice sizes collapse into a single curve when we choose the correlationlength critical exponents $\nu=1.00$. Here periodic boundary conditions are used with $\Delta=0.5$ and $\phi=\pi$.

through the self-duality point $(V=2 J)$. With a finite value of $\Delta$, the transition from the extended phase to the localized phase has to pass through an intermediate phase, and the critical point will develop into a critical region, which is sandwiched between the extended and localized states. Various available methods have been incorporated in identifying quantum critical points (QCPs) from numerical simulations. A useful quantity in characterizing quantum criticality of disordered systems is the inverse participation ratio (IPR), which is equivalent to the second-order participation Rényi entropy. Since there is no mobility edge in the energy spectrum, we then use the mean inverse participation ratio (MIPR) to characterize the degree of the extensivity in space of the wave function in different phases. The MIPR presents a power- 

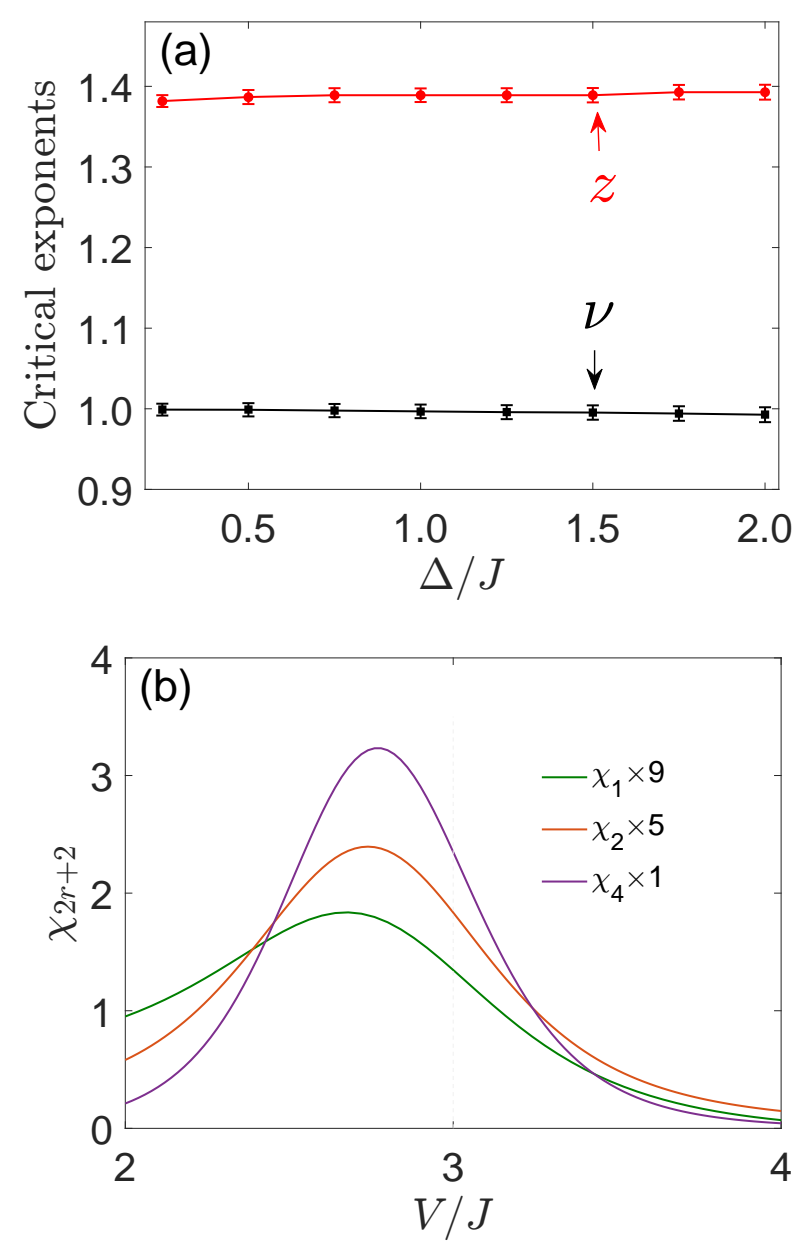

FIG. 7. (a) The fitted values of critical exponents $\nu$ and $z$ as $\Delta$ varies. Here periodic boundary conditions are used with $\phi=\pi$. (b) The GFS $\chi_{2 r+2}$ as a function of the strength of the incommensurate potential $V$ around $V_{c 2}=3$ for a small system size $N=13$. Note that $\chi_{1}$ and $\chi_{2}$ have been respectively increased by a factor of 9 and 5 for guiding the eyes.

law scaling $\propto N^{-d^{*}}$ in distinct phases, where $d^{*}=1$ in the extended phase, $d^{*}=0$ in the localized phase and the exponent $0<d^{*}<1$ in the intermediate critical phase.

We have developed accelerated methods for the location of critical points by the extrema of the universal order parameters. In this context, higher-order GFSs are more efficient in spotlighting the pseudo-critical points, even in the moderately large systems. The enhanced sensitivity is propitious for extracting the associated universal information from the finite-size scaling in quasiperiodic QCPs, whose system sizes are rapidly growing three-subsequence Fibonacci numbers [105]. This distinguishing feature becomes especially crucial in interacting many-body systems and higher-dimensional systems. One can see from Fig. $7(\mathrm{~b})$ that $\chi_{4}$ has already spied on the pseudocritical point for $N=13$ via the visible peak. By performing a detailed numerical simulation, we find different orders of GFS obey power-law scaling in the vicinity of the localization transitions. The single parameter scaling of these macroscopic observables provide selfconsistent results of critical exponents. Moreover, the generalization of fidelity susceptibility poses an efficient avenue to dynamic exponent $z$. The determined values of correlation-length exponent $\nu \simeq 1.000$ and the dynamical exponent $z \simeq 1.388$ suggest that the quantum criticality of localization transitions in the AAH model for $\Delta \neq 0$ lies in a different universality class from the Aubry-André transition $(\Delta=0)$ with $\nu=1.000$, and $z=2.375[32,102]$, where a Aubry-André-type duality may prevent the finite energy excitations from localizing. Understanding the nature of the quasiperiodic localization transition, with and without a finite $p$-wave superconducting pairing, may thereby cut to the heart of the phenomenon. The critical properties of this fixed point are found to be intermediate to the clean and randomly disordered cases. The former case is represented by the clean transverse field Ising model in the celebrated Onsager universality class with $\nu=z=1$, while the latter is symbolized by the Anderson model with $\nu=2 / 3, z=2$ [106].

Last but not the least, another challenge in the study of quasiperiodic models is to separate physically measurable observables from the mathematically intriguing concepts. The quantum metric tensor has been experimentally measured with superconducting qubits [107], coupled qubits in diamond [108], and planar microcavity [109]. Thus, an experimental measurement of the correlation-length exponent $\nu$ and the dynamical exponent $z$ becomes tractable. For instance, $z$ governs the low-temperature behavior of the specific heat $C_{v} \sim T^{-z}$ and can be extracted from the density of states $\rho \sim$ $\epsilon^{1 / z-1}$ or through the Kibble-Zurek mechanism [102]. In this respect, our results can be explored in state-of-theart experimental settings for moderate system sizes. Our tentative approach draws a link between quantum information science and analog quasiperiodic systems without explicit order parameters, and it would be interesting to investigate whether our results can be extended to more complex disordered models.

\section{ACKNOWLEDGMENTS}

This work is supported by the National Natural Science Foundation of China (NSFC) under Grant No. 12174194, the startup fund of Nanjing University of Aeronautics and Astronautics under Grant No. 1008YAH20006, Top-notch Academic Programs Project of Jiangsu Higher Education Institutions (TAPP), and stable supports for basic institute research under Grant No. 190101. 
[1] S. L. Sondhi, S. M. Girvin, J. P. Carini, and D. Shahar, "Continuous quantum phase transitions," Rev. Mod. Phys. 69, 315 (1997).

[2] S. Sachdev, Quantum Phase Transitions (Cambridge, UK, 2000).

[3] B. Shen, Y. Zhang, Y. Komijani, M. Nicklas, R. Borth, A. Wang, Y. Chen, Z. Nie, L. Rui, and L. Xin, "Strangemetal behaviour in a pure ferromagnetic Kondo lattice," Nature 579, 51-55 (2020).

[4] L. Bayha, M. Holten, R. Klemt, K. SubraAmanian, and S. Jochim, "Observing the emergence of a quantum phase transition shell by shell," Nature 587, 583-587 (2020).

[5] M. L. Cai, Z. D. Liu, W. D. Zhao, Y. K. Wu, Q. X. Mei, Y. Jiang, L. He, X. Zhang, Z. C. Zhou, and L. M. Duan, "Observation of a quantum phase transition in the quantum Rabi model with a single trapped ion," Nature Communications 12, 1126 (2021).

[6] M. Liu, S. Chesi, Z. J. Ying, X. Chen, H. G. Luo, and H. Q. Lin, "Universal scaling and critical exponents of the anisotropic quantum Rabi model," Phys. Rev. Lett. 119, 220601 (2017).

[7] M. J. Hwang, R. Puebla, and M. B. Plenio, "Quantum phase transition and universal dynamics in the Rabi model," Phys. Rev. Lett. 115, 180404 (2015).

[8] M. A. Metlitski C. Xu C. Wang, A. Nahum and T. Senthil, "Deconfined quantum critical points: Symmetries and dualities," Phys. Rev. X 7, 031051 (2017).

[9] H. Shao, W. Guo, and A. W. Sandvik, "Quantum criticality with two length scales," Science 352, 213-216 (2016).

[10] Z. Bi, E. Lake, and T. Senthil, "Landau ordering phase transitions beyond the Landau paradigm," Phys. Rev. Research 2, 023031 (2020).

[11] A. I. Goldman and R. F. Kelton, "Quasicrystals and crystalline approximants," Rev. Mod. Phys. 65, 213 (1993).

[12] S. Iyer, V. Oganesyan, G. Refael, and D. A. Huse, "Many-body localization in a quasiperiodic system," Phys. Rev. B 87, 134202 (2013).

[13] M. Kohmoto, B. Sutherland, and C. Tang, "Critical wave functions and a cantor-set spectrum of a onedimensional quasicrystal model," Phys. Rev. B 35, 1020 (1987).

[14] A. Lagendijk, B. V. Tiggelen, and D. S. Wiersma, "Fifty years of Anderson localization," Phys. Today 62, 24 (2009).

[15] Y. V. Kartashov L. Torner V. V. Konotop hen, C. Huang and F. Ye, "Localization and delocalization of light in photonic moiré lattices," Nature 577, 42-46 (2019).

[16] M. Gonalves, B. Amorim, E. V. Castro, and P. Ribeiro, "Hidden dualities in 1D quasiperiodic lattice models," arXiv:2103.03895.

[17] X. Cai, L. J. Lang, S. Chen, and Y. Wang, "Topological superconductor to Anderson localization transition in one-dimensional incommensurate lattices," Phys. Rev. Lett. 110, 176403 (2013).

[18] L. Y. Gong and P. Q. Tong, "Von Neumann entropy of an electron in one-dimensional Determined Potentials," Chin. Phys. Lett. 22, 2759 (2005).
[19] U. Agrawal, S. Gopalakrishnan, and R. Vasseur, "Universality and quantum criticality in quasiperiodic spin chains," Nature Communications 11, 2225 (2020).

[20] S. Roy, T. Mishra, B. Tanatar, and S. Basu, "Reentrant localization transition in a quasiperiodic chain," Phys. Rev. Lett. 126, 106803 (2021).

[21] V. Goblot, A. Strkalj, N. Pernet, J. L. Lado, C. Dorow, A. Lemaître, L. Le Gratiet, A. Harouri, I. Sagnes, S. Ravets, and et al, "Emergence of criticality through a cascade of delocalization transitions in quasiperiodic chains," Nature Physics 16, 832-836 (2020).

[22] Y. Wang, X. Xia, L. Zhang, H. Yao, S. Chen, J. You, Q. Zhou, and X. J. Liu, "One-dimensional quasiperiodic mosaic lattice with exact mobility edges," Phys. Rev. Lett. 125, 196604 (2020).

[23] B. Sutherland, "Localization of electronic wave functions due to local topology," Phys. Rev. B 34, 5208 (1986).

[24] H. Hiramoto and M. Kohmoto, "Electronic spectral and wavefunction properties of one-dimensional quasiperiodic systems: a scaling approach," Int. J. Mod Phys B 6, 281-320 (1992).

[25] H. Hiramoto and M. Kohmoto, "Scaling analysis of quasiperiodic systems: Generalized Harper model," Phys. Rev. B 40, 8225 (1989).

[26] P. Chen S. Liu, T. Wang and X. T. Gao, "Phase diagram of a generalized off-diagonal Aubry-André model with $p$-wave pairing," J. Phys. B:At. Mol. Opt. Phys. 51, 025301 (2017).

[27] Q. B. Zeng, S. Chen, and R. Lü, "Quench dynamics in the Aubry-André-Harper model with $p$-wave superconductivity," New J. Phys. 20, 053012 (2018).

[28] X. Tong, Y. M. Meng, X. Jiang, C. Lee, G. Dias de Moraes Neto, and X. Gao, "Dynamics of a quantum phase transition in the Aubry-André-Harper model with p-wave superconductivity," Phys. Rev. B 103, 104202 (2021).

[29] F. Liu, S. Ghosh, and Y. D. Chong, "Localization and adiabatic pumping in a generalized Aubry-AndréHarper model," Phys. Rev. B 91, 014108 (2015).

[30] A. Dhar A. Purkayastha, S. Sanyal and M. Kulkarni, "Anomalous transport in the Aubry-André-Harper model in isolated and open systems," Phys. Rev. B 97, 174206 (2018).

[31] Q. B. Zeng, S. Chen, and R. Lü, "Generalized AubryAndré-Harper model with $p$-wave superconducting pairing," Phys. Rev. B 94, 125408 (2016).

[32] B. B. Wei, "Fidelity susceptibility in one-dimensional disordered lattice models," Phys. Rev. A 99, 042117 (2019).

[33] N. Roy and A. Sharma, "Study of counterintuitive transport properties in the Aubry-André-Harper model via entanglement entropy and persistent current," Phys. Rev. B 100, 195143 (2019).

[34] J. Fraxanet, U. Bhattacharya, T. Grass, D. Rakshit, M. Lewenstein, and A. Dauphin, "Topological properties of the long-range Kitaev chain with Aubry-AndréHarper modulation," Phys. Rev. Research 3, 013148 (2021).

[35] T. Xiao, D. Z. Xie, Z. L. Dong, T. Chen, W. Yi, and B. Yan, "Observation of topological phase with critical 
localization in a quasi-periodic lattice," Science Bulletin 66, 2175-2180 (2021).

[36] J. Wang, X. J. Liu, X. Gao, and H. Hui, "Phase diagram of a non-abelian Aubry-André-Harper model with p-wave superfluidity," Phys. Rev. B 93, 104504 (2016).

[37] I. Mondragon-Shem and T. L. Hughes, "Signatures of metal-insulator and topological phase transitions in the entanglement of one-dimensional disordered fermions," Phys. Rev. B 90, 104204 (2014).

[38] Y. Yoo, J. Lee, and B. Swingle, "Nonequilibrium steady state phases of the interacting Aubry-André-Harper model," Phys. Rev. B 102, 195142 (2020).

[39] Q. B. Zeng, S. Chen, and R. Lü, "Anderson localization in the non-Hermitian Aubry-André-Harper model with physical gain and loss," Phys. Rev. A 95, 062118 (2017).

[40] X. L. Zhao, Z. C. Shi, C. S. Yu, and X. X. Yi, "Effect of loss on the topological features of dimer chains described by the extended Aubry-André-Harper model," Phys. Rev. A 95, 043837 (2017).

[41] S. Longhi, "Metal-insulator phase transition in a nonHermitian Aubry-André-Harper model," Phys. Rev. B 100, 125157 (2019).

[42] Q. B. Zeng, Y. B. Yang, and Y. Xu, "Topological phases in non-Hermitian Aubry-André-Harper models," Phys. Rev. B 101, 020201 (2020).

[43] S. Longhi, "Phase transitions in a non-Hermitian AubryAndré-Harper model," Phys. Rev. B 103, 054203 (2021).

[44] D. W. Zhang, Y. L. Chen, G. Q. Zhang, L. J. Lang, and S. L. Zhu, "Skin superfluid, topological Mott insulators, and asymmetric dynamics in an interacting nonHermitian Aubry-André-Harper model," Phys. Rev. B 101, 235150 (2020).

[45] T. Liu, H. Guo, Y. Pu, and S. Longhi, "Generalized Aubry-André self-duality and mobility edges in nonHermitian quasiperiodic lattices," Phys. Rev. B 102, 024205 (2020).

[46] S. A. Gredeskul and Yu. S. Kivshar, "Generation of dark solitons in optical fibers," Phys. Rev. Lett. 62, 977 (1989).

[47] D. N. Christodoulides and Y. Silberberg, "Discretizing light behaviour in linear and nonlinear waveguide lattices," Nature 424, 817-823 (2003).

[48] T. Pertsch, U. Peschel, S. Nolte, A. Tünnermann, and H. Bartelt, "Nonlinearity and Disorder in Fiber Arrays," Phys. Rev. Lett. 93, 053901 (2004).

[49] L Dal Negro, C. J. Oton, Z. Gaburro, L. Pavesi, P. Johnson, A. Lagendijk, R. Righini, M. Colocci, and D. S. Wiersma, "Light transport through the band-edge states of Fibonacci quasicrystals," Phys. Rev. Lett. 90, 055501 (2003).

[50] Y. Lahini, R. Pugatch, F. Pozzi, M. Sorel, R. Morandotti, N. Davidson, and Y. Silberberg, "Observation of a localization transition in quasiperiodic photonic lattices," Phys. Rev. Lett. 103, 013901 (2009).

[51] Y. E. Kraus, Y. Lahini, Z. Ringel, M. Verbin, and O. Zilberberg, "Topological states and adiabatic pumping in quasicrystals," Phys. Rev. Lett. 109, 106402 (2012).

[52] G. Roati, C D'Errico, L. Fallani, M. Fattori, C. Fort, M. Zaccanti, G. Modugno, M. Modugno, and M. Inguscio, "Anderson localization of a non-interacting BoseEinstein condensate," Nature 453, 895-898 (2008).

[53] M. Yahyavi, B. Hetényi, and B. Tanatar, "General- ized Aubry-André-Harper model with modulated hopping and p-wave pairing," Phys. Rev. B 100, 064202 (2019).

[54] A. Štrkalj, E. V. H Doggen, I. V. Gornyi, and O. Zilberberg, "Many-body localization in the interpolating Aubry-André-Fibonacci model," Phys. Rev. Research 3, 033257 (2021).

[55] L. Y. Gong and P. Q. Tong, "Fidelity, fidelity susceptibility, and von Neumann entropy to characterize the phase diagram of an extended Harper model," Phys. Rev. B 78, 115114 (2008).

[56] T. Werlang, C. Trippe, G.A.P. Ribeiro, and G. Rigolin, "Quantum correlations in spin chains at finite temperatures and quantum phase transitions," Phys. Rev. Lett. 105, 095702 (2010).

[57] B. B. Wei and X. C. Lv, "Fidelity susceptibility in the quantum Rabi model," Phys. Rev. A 97, 013845 (2018).

[58] S. Garnerone, N. T. Jacobson, S. Haas, and P. Zanardi, "Fidelity approach to the disordered quantum XY model," Phys. Rev. Lett. 102, 057205 (2009).

[59] S. Chen, L. Wang, Y. Hao, and Y. Wang, "Intrinsic relation between ground-state fidelity and the characterization of a quantum phase transition," Phys. Rev. A 77, 032111 (2008).

[60] R. Mao, Y. W. Dai, S. Y. Cho, and H. Q. Zhou, "Quantum coherence and spin nematic to nematic quantum phase transitions in biquadratic spin-1 and spin-2 $X Y$ chains with rhombic single-ion anisotropy," Phys. Rev. B 103, 014446 (2021).

[61] J. J. Kinnunen, Z. Wu, and G. M. Bruun, "Induced pwave pairing in Bose-Fermi mixtures," Phys. Rev. Lett. 121, 253402 (2018).

[62] B. Liu, X.P. Li, Randall G. Hulet, and W. Vincent Liu, "Detecting $\pi$-phase superfluids with $p$-wave symmetry in a quasi-one-dimensional optical lattice," Phys. Rev. A 94, 031602 (2016).

[63] Y. Li, Q. Y. Li, W. Li, T. Liu, and D. Adroja, "Spin dynamics and griffiths singularity in the random quantum Ising magnet prtinbo $_{6}, "$ npj Quantum Mater. 6, 34 (2021).

[64] T. Caneva, R. Fazio, and G. E. Santoro, "Adiabatic quantum dynamics of a random Ising chain across its quantum critical point," Phys. Rev. B 76, 144427 (2007).

[65] K. Machida and M. Fujita, "Quantum energy spectra and one-dimensional quasiperiodic systems," Phys. Rev. B 34, 7367 (1986).

[66] M. Kohmoto, "Metal-insulator transition and scaling for incommensurate systems," Phys. Rev. Lett. 51, 1198 (1983).

[67] F. Evers and A. D. Mirlin, "Anderson transitions," Rev. Mod. Phys. 80, 1355-1417 (2008).

[68] D. C. Licciardello and D. J. Thouless, "Conductivity and mobility edges in disordered systems. II. Further calculations for the square and diamond lattices," J. Phys.C: Solid State Phys 11, 925-936 (1978).

[69] F. Wegner, "Inverse participation ratio in $2+\varepsilon$ dimensions," Z. Phys.B 36, 209-214 (1980).

[70] G. Misguich, V. Pasquier, and J. M. Luck, "Inverse participation ratios in the XXZ spin chain," Phys. Rev. B 94, 155110 (2016).

[71] F. Dukesz, M. Zilbergerts, and L. F. Santos, "Interplay between interaction and (un)correlated disorder in onedimensional many-particle systems: Delocalization and 
global entanglement," New J. Phys. 11, 043026 (2009).

[72] D. J. Thouless, "Electrons in disordered systems and the theory of localization," Phys. Rep. 13, 93-142 (1974).

[73] J. Biddle, D. J. Priour, B. Wang, and S. Das Sarma, "Localization in one-dimensional lattices with nonnearest-neighbor hopping: Generalized Anderson and Aubry-André models," Phys. Rev. B 83, 075105 (2011).

[74] S. Ganeshan, J. H. Pixley, and S. Das Sarma, "Nearest neighbor tight binding models with an exact mobility edge in one dimension," Phys. Rev. Lett. 114, 146601 (2015).

[75] T. Kohlert M. Schreiber P. Bordia X. Li S. Das Sarma H. P. Lüschen, S. Scherg and I. Bloch, "Single-particle mobility edge in a one-dimensional quasiperiodic optical lattice," Phys. Rev. Lett. 120, 160404 (2018).

[76] Q. Guo, C. Cheng, Z. H. Sun, Z. Song, H. Li, Z. Wang, W. Ren, H. Dong, D. Zheng, and Y. R. Zhang, "Observation of energy-resolved many-body localization," Nature Physics 17, 234-239 (2021).

[77] A. Polkovnikov, "Microscopic diagonal entropy and its connection to basic thermodynamic relations," Ann. Phys. 326, 486-499 (2011).

[78] L. F. Santos, A. Polkovnikov, and M. Rigol, "Entropy of isolated quantum systems after a quench," Phys. Rev. Lett. 107, 040601 (2011).

[79] L. Vidmar, L. Hackl, E. Bianchi, and M. Rigol, "Volume law and quantum criticality in the entanglement entropy of excited eigenstates of the quantum Ising model," Phys. Rev. Lett. 121, 220602 (2018).

[80] L. Pastur and V. Slavin, "Area Law scaling for the entropy of disordered quasifree fermions," Phys. Rev. Lett. 113, 150404 (2014).

[81] B. Kramer and A. Mackinnon, "Localization: Theory and experiment," Rep.Prog.Phys 56, 1469-1564 (1993).

[82] G. De Tomasi and I. M. Khaymovich, "Multifractality meets entanglement: Relation for nonergodic extended states," Phys. Rev. Lett. 124, 200602 (2020).

[83] S. Yang, S. J. Gu, C. P. Sun, and H.Q. Lin, "Fidelity susceptibility and long-range correlation in the Kitaev honeycomb model," Phys. Rev. A 78, 012304 (2008).

[84] P. Zanardi and N. Paunkovi, "Ground state overlap and quantum phase transitions," Phys. Rev. E 74, 031123 (2006).

[85] W. L. You, Y. W. Li, and S. J. Gu, "Fidelity, dynamic structure factor, and susceptibility in critical phenomena," Phys. Rev. E 76, 022101 (2007).

[86] M. Thesberg and E. S Sørensen, "General quantum fidelity susceptibilities for the $J_{1}-J_{2}$ chain," Phys. Rev. B 84, 224435 (2011).

[87] Z. Zhu, G. Y. Sun, W. L. You, and D. N. Shi, "Fidelity and criticality of a quantum Ising chain with long-range interactions," Phys. Rev. A 98, 023607 (2018).

[88] J. Ren, W. L. You, and X. Wang, "Entanglement and correlations in a one-dimensional quantum spin$\frac{1}{2}$ chain with anisotropic power-law long-range interactions," Phys. Rev. B 101, 094410 (2020).

[89] G. Y. Sun, B. B. Wei, and S. P. Kou, "Fidelity as a probe for a deconfined quantum critical point," Phys. Rev. B 100, 064427 (2019).

[90] M. Pandey, P. W. Claeys, D. K. Campbell, A. Polkovnikov, and D. Sels, "Adiabatic eigenstate deformations as a sensitive probe for quantum chaos," Phys. Rev. X 10, 041017 (2020).

[91] F. M. Surace, M. Votto, E. G. Lazo, A. Silva, M. Dal- monte, and G. Giudici, "Exact many-body scars and their stability in constrained quantum chains," Phys. Rev. B 103, 104302 (2021).

[92] T. Leblond, D. Sels, A. Polkovnikov, and M. Rigol, "Universality in the onset of quantum chaos in manybody systems," Phys. Rev. B 104, L201117 (2021).

[93] M. Alishahiha and A. F. Astaneh, "Holographic fidelity susceptibility," Phys. Rev. D 96, 086004 (2017).

[94] W. L. You and L. He, "Generalized fidelity susceptibility at phase transitions," J. Phys. Condens. Matter 27, 205601 (2015).

[95] P. Zanardi, P. Giorda, and M. Cozzini, "Informationtheoretic differential geometry of quantum phase transitions," Phys. Rev. Lett. 99, 100603 (2007).

[96] D. Braun, G. Aerardo, F. Benatti, R. Floreanini, U. Marzolino, M. W. Mitchell, and S. Pirandola, "Quantum-enhanced measurements without entanglement," Rev. Mod. Phys. 90, 035006 (2018).

[97] M. E. Fisher and M. N. Barber, "Scaling theory for finite-size effects in the critical region," Phys. Rev. Lett. 28, 1516 (1972).

[98] M. Roncaglia, L. Campos Venuti, and E. Degli, "Finding critical points using improved scaling ansätze," J. Stat. Mech. Theory Exp. 2015, P04005 (2015).

[99] A. Osterloh, L. Amico, G. Falci, and R. Fazio, "Scaling of entanglement close to a quantum phase transition," Nature 416, 608-610 (2002).

[100] J. Ren, Y. Wang, and W. L. You, "Quantum phase transitions in spin-1 XXZ chains with rhombic singleion anisotropy," Phys. Rev. A 97, 042318 (2018).

[101] A. F. Albuquerque, F. Alet, C. Sire, and S. Capponi, "Quantum critical scaling of fidelity susceptibility," Phys. Rev. B 81, 064418 (2010).

[102] A. Sinha, M. M. Rams, and J. Dziarmaga, "KibbleZurek mechanism with a single particle: Dynamics of the localization-delocalization transition in the AubryAndré model," Phys. Rev. B 99, 094203 (2019).

[103] A. Altland and M. R. Zirnbauer, "Nonstandard symmetry classes in mesoscopic normal-superconducting hybrid structures," Phys. Rev. B 55, 1142 (1997).

[104] A. B. Harris, "Effect of random defects on the critical behaviour of ising models," J. Phys. C: Solid State Phys 7, 1671 (1974).

[105] T. Cookmeyer, J. Motruk, and J. E. Moore, "Critical properties of the ground-state localizationdelocalization transition in the many-particle AubryAndré model," Phys. Rev. B 101, 174203 (2020).

[106] J. C. C. Cestari, A. Foerster, M. A. Gusmão, and M. Continentino, "Critical exponents of the disorder-driven superfluid-insulator transition in onedimensional Bose-Einstein condensates," Phys. Rev. A 84, 055601 (2011).

[107] X. Tan, D. W. Zhang, Z. Yang, J. Chu, Y. Q. Zhu, D. Li, X. Yang, S. Song, Z. Han, Z. Li, Y. Dong, H. F. Yu, H. Yan, S. L. Zhu, and Y. Yu, "Experimental measurement of the quantum metric tensor and related topological phase transition with a superconducting qubit," Phys. Rev. Lett. 122, 210401 (2019).

[108] M. Yu, P. Yang, M. Gong, Q. Cao, Q. Lu, H. Liu, M. B. Plenio, F. Jelezko, T. Ozawa, and N. Goldman, "Experimental measurement of the quantum geometric tensor using coupled qubits in diamond," Natl. Sci. Rev. 7, 254-260 (2020).

[109] A. Gianfrate, O. Bleu, L. Dominici, V. Ardizzone, M. De 
Giorgi, D. Ballarini, G. Lerario, K. W. West, L. N. Pfeiffer, D. D. Solnyshkov, D. Sanvitto, and G. Malpuech,
"Measurement of the quantum geometric tensor and of the anomalous Hall drift," Nature 578, 381-385 (2020). 